LINEAR AND NON-LINEAR THEORY OF

GENERALIZED FUNCTIONS AND ITS APPLICATIONS

BANACH CENTER PUBLICATIONS, VOLUME 88

INSTITUTE OF MATHEMATICS

POLISH ACADEMY OF SCIENCES

WARSZAWA 2010

\title{
CHARACTERIZATION OF SURJECTIVE CONVOLUTION OPERATORS ON SATO'S HYPERFUNCTIONS
}

\author{
MICHAEL LANGENBRUCH \\ Department of Mathematics \\ University of Oldenburg \\ 26111 Oldenburg, Germany \\ E-mail: michael.langenbruch@uni-oldenburg.de
}

\begin{abstract}
Let $\mu \in \mathcal{A}\left(\mathbb{R}^{d}\right)^{\prime}$ be an analytic functional and let $T_{\mu}$ be the corresponding convolution operator on Sato's space $\mathcal{B}\left(\mathbb{R}^{d}\right)$ of hyperfunctions. We show that $T_{\mu}$ is surjective iff $T_{\mu}$ admits an elementary solution in $\mathcal{B}\left(\mathbb{R}^{d}\right)$ iff the Fourier transform $\widehat{\mu}$ satisfies Kawai's slowly decreasing condition $(S)$. We also show that there are $0 \neq \mu \in \mathcal{A}\left(\mathbb{R}^{d}\right)^{\prime}$ such that $T_{\mu}$ is not surjective on $\mathcal{B}\left(\mathbb{R}^{d}\right)$.
\end{abstract}

1. Introduction. Surjectivity of convolution operators has been characterized in many classical spaces of (generalized) functions including spaces of real analytic and holomorphic functions, spaces of (ultra)differentiable functions and spaces of (ultra)distributions and Fourier hyperfunctions, respectively. A selection of corresponding papers is contained in the references and it is intended only as a first hint towards the corresponding literature (see $1,7,9,11,16,18,20$ ).

For convolution operators on Sato's space $\mathcal{B}\left(\mathbb{R}^{d}\right)$ of hyperfunctions however, a characterization of surjectivity seems to be missing. One reason for this might be that $\mathcal{B}\left(\mathbb{R}^{d}\right)$ does not admit a suitable topology and hence topological methods are not directly applicable.

A sufficient condition for the surjectivity of $T_{\mu}$ on $\mathcal{B}\left(\mathbb{R}^{d}\right)$ is Kawai's slowly decreasing condition $(S)$ (see [11] and (1) below). Using Kawai's condition, Okada 18] proved that $T_{\mu}$ is surjective on $\mathcal{B}\left(\mathbb{R}^{d}\right)$ for any ultradistribution $\mu \neq 0$ with compact support. Moreover, $(S)$ can also be used to show that $T_{\mu}$ is surjective on $\mathcal{B}\left(\mathbb{R}^{d}\right)$ for any $0 \neq \mu \in \mathcal{A}(\{0\})^{\prime}$ (see $[18]$, while the present paper shows that there are $0 \neq \mu \in \mathcal{A}\left(\mathbb{R}^{d}\right)^{\prime}$ such that $T_{\mu}$

2000 Mathematics Subject Classification: Primary 46F15; Secondary 46N20, 44A35.

Key words and phrases: hyperfunctions, convolution operators, surjectivity.

The paper is in final form and no version of it will be published elsewhere. 
is not surjective on $\mathcal{B}\left(\mathbb{R}^{d}\right)$. In fact, we will show that $(S)$ is also a necessary condition for surjectivity and we will clarify the connection of surjectivity and the existence of elementary solutions for $T_{\mu}$. More precisely we will prove the following

Main Theorem. For $\mu \in \mathcal{A}\left(\mathbb{R}^{d}\right)^{\prime}$ the following are equivalent:

a) $T_{\mu}: \mathcal{B}\left(\mathbb{R}^{d}\right) \rightarrow \mathcal{B}\left(\mathbb{R}^{d}\right)$ is surjective.

b) $T_{\mu}$ admits an elementary solution $E \in \mathcal{B}\left(\mathbb{R}^{d}\right)$.

c) $\widehat{\mu}$ satisfies the following condition $(S)$ : For any $\delta>0$ there is $C>0$ such that for any $t \in \mathbb{R}^{d}$ with $|t| \geq C$ there is $\zeta \in \mathbb{C}^{d}$ such that

$$
|\zeta-t| \leq \delta|t| \quad \text { and } \quad|\widehat{\mu}(\zeta)| \geq e^{-\delta|\zeta|} .
$$

Recall that $E \in \mathcal{B}\left(\mathbb{R}^{d}\right)$ is an elementary solution for $T_{\mu}$ if $T_{\mu}(E)=\delta$, where $\delta$ denotes Dirac's $\delta$-distribution.

Notice that we do not need a condition on the location of zeroes of $\widehat{\mu}$ for the characterization in our Main Theorem. This is different from the characterization of surjective convolution operators on Fourier hyperfunctions and on modified Fourier hyperfunctions (see 13 and 20$]$ ).

The implication "c) $\Rightarrow$ a)" was proved in 11 using convolution operators on holomorphic functions defined on tube domains. We will give a different proof here which is based on the space $\mathcal{R}\left(\mathbb{D}^{d}\right)$ of modified Fourier hyperfunctions (see 2.1 and 3.3). This space is also the main tool when proving that b) implies c) (see 3.1).

Using Baire's category theorem we then show that there are $0 \neq \mu \in \mathcal{A}\left(\mathbb{R}^{d}\right)^{\prime}$ such that $T_{\mu}$ is not surjective on $\mathcal{B}\left(\mathbb{R}^{d}\right)$ (see 3.4$)$.

2. Preliminaries. We will recall some basic notions and results concerning hyperfunctions and modified Fourier hyperfunctions in this section (see [1], [10] and [19] for a systematic study) and then show how topological methods can be used to characterize surjectivity of convolution operators on $\mathcal{B}\left(\mathbb{R}^{d}\right)$ (see 2.1 ).

Hyperfunctions may be defined as boundary values of holomorphic functions as follows: let $\mathbb{C}_{\sharp}^{d}:=\left\{z \in \mathbb{C}^{d} \mid \Im\left(z_{l}\right) \neq 0\right.$ for any $\left.l\right\}$ and $\mathbb{C}_{\sharp, j}^{d}:=\left\{z \in \mathbb{C}^{d} \mid \Im\left(z_{l}\right) \neq 0\right.$ for any $l \neq j\}$. Then the space of hyperfunctions is given by

$$
\mathcal{B}\left(\mathbb{R}^{d}\right):=\mathcal{H}\left(\mathbb{C}_{\sharp}^{d}\right) /\left(\sum_{j \leq d} \mathcal{H}\left(\mathbb{C}_{\sharp, j}^{d}\right)\right) .
$$

We always assume in this paper that $\mu \in \mathcal{A}\left(\mathbb{R}^{d}\right)^{\prime}$ is fixed. $\widehat{\mu}$ then is an entire function and there is $K$ such that for any $\varepsilon>0$ there is $C_{\varepsilon}$ such that

$$
|\widehat{\mu}(z)| \leq C_{\varepsilon} e^{\varepsilon|z|+K|\Im(z)|} .
$$

For $u \in \mathcal{H}\left(\mathbb{C}_{\sharp}^{d}\right)$ let

$$
\mu * u(z):=\langle\xi \mu, u(z-\xi)\rangle \quad \text { if } z \in \mathbb{C}_{\sharp}^{d} .
$$

The convolution operator $T_{\mu}$ on $\mathcal{B}\left(\mathbb{R}^{d}\right)$ is then defined by

$$
T_{\mu}([u]):=[\mu * u] \quad \text { if }[u] \in \mathcal{B}\left(\mathbb{R}^{d}\right) .
$$


In the following, we will usually write $u \in \mathcal{B}\left(\mathbb{R}^{d}\right)$ and we will thus not distinguish in notation between the defining function and its equivalence class in $\mathcal{B}\left(\mathbb{R}^{d}\right)$.

Similarly, the space $\mathcal{R}\left(\mathbb{D}^{d}\right)$ of modified Fourier hyperfunctions on the radial compactification $\mathbb{D}^{d}$ of $\mathbb{R}^{d}$ may be defined as a space of boundary values using in (2) spaces of exponentially increasing functions defined as follows (see $[19$, p. $257 \mathrm{ff}]$ ): let $\mathbb{Y}^{d}$ be the radial compactification of $\mathbb{C}^{d}=\mathbb{R}^{2 d}$. For $W$ open in $\mathbb{Y}^{d}$ let

$$
\mathcal{O}_{i n c}(W):=\left\{f \in \mathcal{H}\left(\mathbb{C}^{d} \cap W\right)\left|\sup _{Z}\right| f(z) \mid e^{-|z| / j}<\infty \text { for any } Z \Subset W \text { and any } j\right\}
$$

where $Z \Subset W$ means that $Z$ is relatively compact in $W$ (and open). Let $U:=\left\{z \in \mathbb{C}^{d} \mid\right.$ $\left.|\Im(z)|<\left(1+|\Re(z)|^{2}\right)^{1 / 2} / 2\right\}, V:=(\bar{U})^{\circ} \subset \mathbb{Y}^{d}, V_{\sharp}:=\left\{z \in V \mid \Im\left(z_{l}\right) \neq 0\right.$ for any $\left.l\right\}$ and $V_{\sharp, j}:=\left\{z \in V \mid \Im\left(z_{l}\right) \neq 0\right.$ for any $\left.l \neq j\right\}$. The modified Fourier hyperfunctions on $\mathbb{R}^{d}$ are defined by

$$
\mathcal{R}\left(\mathbb{D}^{d}\right):=\mathcal{O}_{i n c}\left(V_{\sharp}\right) /\left(\sum_{j \leq d} \mathcal{O}_{i n c}\left(V_{\sharp, j}\right)\right) .
$$

Alternatively, $\mathcal{R}\left(\mathbb{D}^{d}\right)$ may be defined by duality (see 19 , Thm. 4.25]), namely as the dual space $\widetilde{P}_{*}\left(\mathbb{D}^{d}\right)^{\prime}$ of $\widetilde{P}_{*}\left(\mathbb{D}^{d}\right):=\operatorname{limind}_{j \rightarrow \infty} \widetilde{P}_{*, j}$, where

$$
\widetilde{P}_{*, j}:=\left\{f \in \mathcal{H}\left(W_{j}\right)\left|\|f\|_{j}:=\sup _{z \in W_{j}}\right| f(z) \mid \exp (|z| / j)<\infty\right\}
$$

for $W_{j}:=\left\{z \in \mathbb{C}^{d}|| \Im(z) \mid<\left(\underset{1}{1}+|\Re(z)|^{2}\right)^{1 / 2} / j\right\}$. The duality of $\mathcal{R}\left(\mathbb{D}^{d}\right)$ and $\widetilde{P}_{*}\left(\mathbb{D}^{d}\right)$ is defined for $u \in \mathcal{R}\left(\mathbb{D}^{d}\right)$ and $f \in \widetilde{P}_{*}\left(\mathbb{D}^{d}\right)$ by

$$
(u, f)=\sum_{\sigma \in\{1,-1\}^{d}} \operatorname{sign}(\sigma) \int_{\gamma_{j, \sigma}} u(z) f(z) d z
$$

where $\gamma_{j, \sigma}$ is the path defined by $x+i e_{\sigma}\left(1+|x|^{2}\right)^{1 / 2} / j$ for a unit vector $e_{\sigma}$ in the corresponding orthant and large $j$. By (6) and (2), the restriction

$$
\mid \mathbb{R}^{d}: \mathcal{R}\left(\mathbb{D}^{d}\right) \rightarrow \mathcal{B}\left(\mathbb{R}^{d}\right) \text { is well defined and }\left.\mathcal{R}\left(\mathbb{D}^{d}\right)\right|_{\mathbb{R}^{d}}=\mathcal{B}\left(\mathbb{R}^{d}\right)
$$

(see [19, sect. 1.2 i)]). By [19, 3.2.3] we have

$$
\operatorname{ker}\left(\left.\right|_{\mathbb{R}^{d}}\right)=\mathcal{R}\left(\mathbb{D}^{d} \backslash \mathbb{R}^{d}\right)=\widetilde{P}_{*}\left(\mathbb{D}^{d} \backslash \mathbb{R}^{d}\right)^{\prime}
$$

where $\widetilde{P}_{*}\left(\mathbb{D}^{d} \backslash \mathbb{R}^{d}\right):=\operatorname{limind} \operatorname{in}_{j \rightarrow \infty} \widetilde{P}_{*, \infty, j}$ for

$$
\widetilde{P}_{*, \infty, j}:=\left\{f \in \mathcal{H}\left(W_{\infty, j}\right)\left|\|f\|_{\infty, j}:=\sup _{z \in W_{\infty, j}}\right| f(z) \mid \exp (|z| / j)<\infty\right\}
$$

where $W_{\infty, j}:=\left\{z \in W_{j}|| z \mid>j\right\}$.

We may define $T_{\mu}(u)$ for $u \in \mathcal{R}\left(\mathbb{D}^{d}\right)$ by using $(4)$ and $(5)$. Then $T_{\mu}(u) \in \mathcal{R}\left(\mathbb{D}^{d}\right)$ and we have

$$
\left.T_{\mu}(u)\right|_{\mathbb{R}^{d}}=T_{\mu}\left(\left.u\right|_{\mathbb{R}^{d}}\right) \quad \text { if } u \in \mathcal{R}\left(\mathbb{D}^{d}\right)
$$


For $f \in \widetilde{P}_{*}\left(\mathbb{D}^{d}\right)$ and large $j$ we get by $(7)$

$$
\begin{aligned}
\left(T_{\mu}(u), f\right) & =\sum_{\sigma \in\{1,-1\}^{d}} \operatorname{sign}(\sigma)\left\langle\xi \mu, \int_{\gamma_{j, \sigma}} u(z-\xi) f(z) d z\right\rangle \\
& =\sum_{\sigma \in\{1,-1\}^{d}} \operatorname{sign}(\sigma)\left\langle\xi \mu, \int_{\widetilde{\gamma}_{j, \sigma}} u(z) f(z+\xi) d z\right\rangle \\
& =\sum_{\sigma \in\{1,-1\}^{d}} \operatorname{sign}(\sigma)\left\langle\xi \mu, \int_{\gamma_{j, \sigma}} u(z) f(z+\xi) d z\right\rangle=(u, \check{\mu} * f) \quad \text { if } u \in \mathcal{R}\left(\mathbb{D}^{d}\right)
\end{aligned}
$$

where $\widetilde{\gamma}_{j, \sigma}$ is defined by $x+i e_{\sigma}\left(1+|x|^{2}\right)^{1 / 2} / j-\xi$ and $j$ is large.

We finally notice that the Fourier transform

$$
\widehat{f}(z):=\int f(x) e^{-i\langle x, z\rangle} d x, \quad f \in \widetilde{P}_{*}\left(\mathbb{D}^{d}\right),
$$

defines a topological isomorphism in $\widetilde{P}_{*}\left(\mathbb{D}^{d}\right)$ (and hence in $\mathcal{R}\left(\mathbb{D}^{d}\right)$ by duality, see 19 ). Moreover,

$$
\widehat{\check{\mu} * f}=\widehat{\tilde{\mu}} \widehat{f} \quad \text { if } f \in \widetilde{P}_{*}\left(\mathbb{D}^{d}\right) .
$$

Lemma 2.1. Let $\mu \in \mathcal{A}\left(\mathbb{R}^{d}\right)^{\prime}$. Then the following are equivalent:

a) $T_{\mu}$ is surjective on $\mathcal{B}\left(\mathbb{R}^{d}\right)$.

b) The mapping

$$
S: \mathcal{R}\left(\mathbb{D}^{d}\right) \times \widetilde{P}_{*}\left(\mathbb{D}^{d} \backslash \mathbb{R}^{d}\right)^{\prime} \rightarrow \mathcal{R}\left(\mathbb{D}^{d}\right), \quad S(\nu, \eta):=T_{\mu}(\nu)-\eta,
$$

is surjective.

c) $B$ is bounded in $\widetilde{P}_{*}\left(\mathbb{D}^{d}\right)$ if $B$ is bounded in $\widetilde{P}_{*}\left(\mathbb{D}^{d} \backslash \mathbb{R}^{d}\right)$ and $\widehat{\mu} \widehat{B}$ is bounded in $\widetilde{P}_{*}\left(\mathbb{D}^{d}\right)$. Proof. "a) $\Rightarrow$ b)" For $\kappa \in \mathcal{R}\left(\mathbb{D}^{d}\right)$ there is $\nu \in \mathcal{B}\left(\mathbb{R}^{d}\right)$ by a) such that $T_{\mu}(\nu)=\left.\kappa\right|_{\mathbb{R}^{d}}$. By (8) there is $\bar{\nu} \in \mathcal{R}\left(\mathbb{D}^{d}\right)$ such that $\left.\bar{\nu}\right|_{\mathbb{R}^{d}}=\nu$. Then

$$
\left.T_{\mu}(\bar{\nu})\right|_{\mathbb{R}^{d}}=T_{\mu}\left(\left.\bar{\nu}\right|_{\mathbb{R}^{d}}\right)=T_{\mu}(\nu)=\left.\kappa\right|_{\mathbb{R}^{d}}
$$

by 10 and hence $S(\bar{\nu}, h)=\kappa$ for $T_{\mu}(\bar{\nu})-\kappa=: h \in \widetilde{P}_{*}\left(\mathbb{D}^{d} \backslash \mathbb{R}^{d}\right)^{\prime}$ by $(9)$.

"b) $\Rightarrow$ a)" For $u \in \mathcal{B}\left(\mathbb{R}^{d}\right)$ there is $\bar{u} \in \mathcal{R}\left(\mathbb{D}^{d}\right)$ such that $\left.\bar{u}\right|_{\mathbb{R}^{d}}=u$ by (8). By assumption we can find $(\nu, h) \in \mathcal{R}\left(\mathbb{D}^{d}\right) \times \widetilde{P}_{*}\left(\mathbb{D}^{d} \backslash \mathbb{R}^{d}\right)^{\prime}$ such that $S(\nu, h)=\bar{u}$ and hence

$$
u=\left.\bar{u}\right|_{\mathbb{R}^{d}}=\left.T_{\mu}(\nu)\right|_{\mathbb{R}^{d}}-\left.h\right|_{\mathbb{R}^{d}}=T_{\mu}\left(\left.\nu\right|_{\mathbb{R}^{d}}\right)
$$

by (9) and (10). This shows a).

$" \mathrm{~b}) \Leftrightarrow \mathrm{c})$ " Since $\mathcal{R}\left(\mathbb{D}^{d}\right)=\widetilde{P}_{*}\left(\mathbb{D}^{d}\right)_{b}^{\prime}$ and $\mathcal{R}\left(\mathbb{D}^{d} \backslash \mathbb{R}^{d}\right)=\widetilde{P}_{*}\left(\mathbb{D}^{d} \backslash \mathbb{R}^{d}\right)_{b}^{\prime}$ are $(F S)$-spaces, $S$ is surjective if and only if ${ }^{t} S: \widetilde{P}_{*}\left(\mathbb{D}^{d}\right) \rightarrow \widetilde{P}_{*}\left(\mathbb{D}^{d}\right) \times \widetilde{P}_{*}\left(\mathbb{D}^{d} \backslash \mathbb{R}^{d}\right)$ is injective with closed range if and only if $B$ is bounded in $\widetilde{P}_{*}\left(\mathbb{D}^{d}\right)$ if ${ }^{t} S(B)$ is bounded in $\widetilde{P}_{*}\left(\mathbb{D}^{d}\right) \times \widetilde{P}_{*}\left(\mathbb{D}^{d} \backslash \mathbb{R}^{d}\right)$ (see 17, section 26]). Since ${ }^{t} S(f)=(\check{\mu} * f,-f)$ for $f \in \widetilde{P}_{*}\left(\mathbb{D}^{d}\right)$ by $[11$ and since the Fourier transformation is a topological isomorphism this proves the claim by (12).

3. The main results. In this section we always denote $\mu \in \mathcal{A}\left(\mathbb{R}^{d}\right)^{\prime}$. We will prove the Main Theorem by means of two lemmata starting with the implication "b) $\Rightarrow c$ )":

LEMMA 3.1. $\widehat{\mu}$ satisfies $(S)$ if $T_{\mu}$ admits an elementary solution $E \in \mathcal{B}\left(\mathbb{R}^{d}\right)$. 
Proof. Let $\bar{E} \in \mathcal{R}\left(\mathbb{D}^{d}\right)$ be an extension of $E$ existing by (8). Then we have by 100

$$
\left.T_{\mu}(\bar{E})\right|_{\mathbb{R}^{d}}=T_{\mu}(E)=\delta
$$

and hence there is $H \in \widetilde{P}_{*}\left(\mathbb{D}^{d} \backslash \mathbb{R}^{d}\right)^{\prime}$ by $(9)$ such that

$$
\delta=T_{\mu}(\bar{E})+H .
$$

If $(S)$ is not true there is $0<\delta \leq 1$ such that for any $l \in \mathbb{N}$ there is $t_{l} \in \mathbb{R}^{d}$ with $\left|t_{l}\right| \geq l$ such that

$$
|\widehat{\mu}(-\zeta)| \leq e^{-\delta|\zeta|} \quad \text { if }\left|\zeta-t_{l}\right| \leq \delta\left|t_{l}\right| .
$$

For $\eta, \zeta \in \mathbb{C}^{d}$ let $\langle\eta, \zeta\rangle:=\sum \eta_{k} \zeta_{k}$ and set

$$
g_{l}(z):=\left(2 \pi /\left(\delta\left|t_{l}\right|\right)\right)^{d / 2} e^{-\left\langle z-t_{l}, z-t_{l}\right\rangle /\left(2 \delta\left|t_{l}\right|\right)}, l \in \mathbb{N},
$$

and notice that $g_{l} \in \widetilde{P}_{*}\left(\mathbb{D}^{d}\right)$ and

$$
g_{l}=\widehat{f}_{l} \quad \text { for } f_{l}(z):=e^{i\left\langle z, t_{l}\right\rangle-\langle z, z\rangle \delta\left|t_{l}\right| / 2} \in \widetilde{P}_{*}\left(\mathbb{D}^{d}\right) .
$$

Since $\bar{E} \in \mathcal{R}\left(\mathbb{D}^{d}\right)=\widetilde{P}_{*}\left(\mathbb{D}^{d}\right)^{\prime}$ and $H \in \widetilde{P}_{*}\left(\mathbb{D}^{d} \backslash \mathbb{R}^{d}\right)^{\prime}$, for any $j \in \mathbb{N}$ there is $C_{1}$ such that by (13), 111 and the Fourier inversion formula

$$
\begin{aligned}
1 & =f_{l}(0)=\left|\left\langle T_{\mu}(\bar{E})+H, f_{l}\right\rangle\right|=\left|(2 \pi)^{-d}\left\langle\widehat{\widehat{E}}, \widehat{\tilde{\mu}} g_{l}\right\rangle+\left\langle H, f_{l}\right\rangle\right| \\
& \leq C_{1}\left(\left\|\widehat{\tilde{\mu}} g_{l}\right\|_{j}+\left\|f_{l}\right\|_{\infty, j}\right) \quad \text { for any } l \in \mathbb{N} .
\end{aligned}
$$

We will show however that both terms of the right hand side of 15 tend to 0 as $l \rightarrow \infty$ if $j$ is large:

I) If $\left|z-t_{l}\right| \leq \delta\left|t_{l}\right| / 2$ then

$$
|\Im(z)| \leq\left|z-t_{l}\right| \leq \delta\left|t_{l}\right| / 2 \quad \text { and } \quad 2\left|t_{l}\right| \geq|z| \geq(1-\delta / 2)\left|t_{l}\right| \geq\left|t_{l}\right| / 2
$$

since $\delta \leq 1$. We thus get for large $l$ by 14

$$
\begin{aligned}
\left|\widehat{\mu}(-z) g_{l}(z)\right| e^{|z| / j} & \leq C_{2} e^{(-\delta+1 / j)|z|+\Im(z)^{2} /\left(2\left|t_{l}\right| \delta\right)} \\
& \leq C_{2} e^{-\delta|z| / 2+\delta\left|t_{l}\right| / 8} \leq C_{2} e^{-\delta\left|t_{l}\right| / 8}
\end{aligned}
$$

if $j \geq 2 / \delta$.

II) Let $z \in W_{j}$ and $|\Re(z)| \geq 1$ (and hence $|\Im(z)| \leq 2|\Re(z)| / j$ ). If $\left|z-t_{l}\right| \geq \delta\left|t_{l}\right| / 2$ we get by (3) (for $\varepsilon:=1 / j$ )

$$
\begin{aligned}
\left|\widehat{\mu}(-z) g_{l}(z)\right| e^{|z| / j} & \leq C_{3} e^{K|\Im(z)|+2|z| / j+|\Im(z)|^{2} /\left(\left|t_{l}\right| \delta\right)-\left|z-t_{l}\right|^{2} /\left(2 \delta\left|t_{l}\right|\right)} \\
& \leq C_{3} e^{2(K+1)|z| / j+4|z|^{2} /\left(j^{2}\left|t_{l}\right| \delta\right)-\left|z-t_{l}\right|^{2} /\left(2 \delta\left|t_{l}\right|\right)} \\
& \leq C_{3} e^{(2 K+2+8 /(j \delta))\left|t_{l}\right| / j+2(K+1)\left|z-t_{l}\right| / j+\left(8 j^{-2}-1 / 2\right)\left|z-t_{l}\right|^{2} /\left(\left|t_{l}\right| \delta\right)} \\
& \leq C_{3} e^{(2 K+2+8 /(j \delta))\left|t_{l}\right| / j+2(K+1)\left|z-t_{l}\right| / j-\left|z-t_{l}\right|^{2} /\left(4\left|t_{l}\right| \delta\right)} \\
& \leq C_{3} e^{\left[(2+\delta)(K+1) / j+8 /\left(j^{2} \delta\right)-\delta / 16\right]\left|t_{l}\right|} \leq C_{3} e^{-\delta\left|t_{l}\right| / 32}
\end{aligned}
$$

if $j$ is large. If $z \in W_{j}$ and $|\Re(z)| \leq 1$ then $|z| \leq 2$ and the right hand side of 16 tends to 0 as $l \rightarrow \infty$. We have thus shown that $\left\|\widehat{\tilde{\mu}} g_{l}\right\|_{j} \rightarrow 0$ as $l \rightarrow \infty$ if $j$ is large. 
III) Let $z \in W_{\infty, j}$ (and hence $j \leq 2|\Re(z)|$ and $|\Im(z)| \leq 2|\Re(z)| / j$ if $j$ is large). Then

$$
\begin{aligned}
\left|f_{l}(z)\right| & \leq e^{\left[|\Im(z)|+|\Im(z)|^{2} \delta / 2-|\Re(z)|^{2} \delta / 2\right]\left|t_{l}\right|} \\
& \leq e^{\left[2|\Re(z)| / j+\left(4 / j^{2}-1 / 2\right) \delta|\Re(z)|^{2}\right]\left|t_{l}\right|} \\
& \leq C_{4} e^{-j^{2} \delta\left|t_{l}\right| / 8} \quad \text { if } j \text { is large. }
\end{aligned}
$$

This shows that $\left\|f_{l}\right\|_{\infty, j} \rightarrow 0$ if $j$ is large.

To show that $(S)$ is sufficient for the surjectivity of $T_{\mu}$ we need the following variant of Harnack's inequality (see [7,3.1]):

LemMA 3.2. Let $F(z), G(z)$ and $F(z) / G(z)$ be holomorphic when $|z|<R, z \in \mathbb{C}^{d}$. For $|z|<R$ we then get

$$
|F(z) / G(z)| \leq \sup _{|\eta|<R}|F(\eta)|\left(\sup _{|\eta|<R}|G(\eta)|\right)^{2|z| /(R-|z|)}|G(0)|^{-(R+|z|) /(R-|z|)} .
$$

Lemma 3.3. $T_{\mu}$ is surjective in $\mathcal{B}\left(\mathbb{R}^{d}\right)$ if $\widehat{\mu}$ satisfies $(S)$.

Proof. a) We will show the criterion of 2.1c). Let $B \subset \widetilde{P}_{*}\left(\mathbb{D}^{d}\right)$ such that $B$ is bounded in $\widetilde{P}_{*}\left(\mathbb{D}^{d} \backslash \mathbb{R}^{d}\right)$ and $\widehat{\mu} \widehat{B}$ is bounded in $\widetilde{P}_{*}\left(\mathbb{D}^{d}\right)$. Since $\widetilde{P}_{*}\left(\mathbb{D}^{d}\right)$ is a compact injective inductive spectrum (and hence regular), there is $j_{1}$ such that any germ $g=\widehat{\mu} \widehat{f} \in \widehat{\mu} \widehat{B}$ has a holomorphic extension $g_{1} \in \mathcal{H}\left(W_{j_{1}}\right)$ contained in a bounded set in $\widetilde{P}_{*, j_{1}}$. Since $B$ is bounded in $\widetilde{P}_{*}\left(\mathbb{D}^{d} \backslash \mathbb{R}^{d}\right.$ ) (which is also a regular inductive spectrum) there is $j_{2}$ such that $\left.B\right|_{W_{j_{2}}} \subset \widetilde{P}_{*, \infty, j_{2}}$ and therefore there is $j_{3}$ such that $\widehat{f}$ has an extension $f_{1} \in \mathcal{H}\left(W_{j_{3}}\right)$ for any $f \in B$ (see the proof of $[19,4.1 .2]$ ). We may assume that $j_{1}=j_{3}$. By the identity theorem this implies that any germ $g=\widehat{\mu} \widehat{f} \in \widehat{\mu} \widehat{B}$ has a holomorphic extension $g_{1}=\widehat{\mu} f_{1}$ contained in a bounded set in $\widetilde{P}_{*, j_{1}}$ where $f_{1} \in \mathcal{H}\left(W_{j_{1}}\right)$. Condition $(S)$ and Lemma 3.2 now easily imply that $\widehat{B}$ (and hence $B$ ) is bounded in $\widetilde{P}_{*}\left(\mathbb{D}^{d}\right)$. The argument is included for the convenience of the reader:

Fix $t \in \mathbb{R}^{d}$ with large $|t|$ and choose $\zeta$ for $\delta=1 /\left(8 j_{2}\right)$ by $(S)$ where $j_{2} \geq j_{1}$ is to be determined later. Then $F:=f_{1}(\zeta+\cdot) \widehat{\mu}(\zeta+\cdot)$ and $G:=\widehat{\mu}(\zeta+\cdot)$ satisfy the assumptions of 3.2 for $R:=|t| /\left(4 j_{2}\right)$. If $|\xi-t| \leq|t| /\left(16 j_{2}\right)$ then $z_{0}:=\xi-\zeta$ satisfies

$$
\left|z_{0}\right| \leq 3|t| /\left(16 j_{2}\right) \text { and } \frac{R+\left|z_{0}\right|}{R-\left|z_{0}\right|} \leq 7 \text { and } \frac{2\left|z_{0}\right|}{R-\left|z_{0}\right|} \leq 6
$$

and by 3.2 and (3) we thus get $C_{1}$ and $C_{2}$ (independent of $j_{2}$ ) such that

$$
\left|f_{1}(\xi)\right|=\left|f_{1}\left(\zeta+z_{0}\right)\right| \leq C_{1} e^{-|\xi| /\left(4 j_{1}\right)+C_{2}|\xi| / j_{2}} \leq C_{1} e^{-|\xi| /\left(8 j_{1}\right)} \quad \text { if } j_{2} \geq 8 C_{2} j_{1}
$$

since $\widehat{\mu} f_{1}$ is contained in a bounded set in $\widetilde{P}_{*, j_{1}}$. This proves the claim.

Combining Lemma 3.1 and Lemma 3.3 we have proved the Main Theorem since it is evident that a) implies b).

It is known that $(S)$ is satisfied for all $0 \neq \mu \in \mathcal{A}(\{0\})^{\prime}$ and for all ultradistributions $\mu \neq 0$ with compact support (see 18$]$ ). For $\mu \in \mathcal{A}\left(\mathbb{R}^{d}\right)^{\prime}$ however this does not hold as we will prove now:

TheOREM 3.4. For any $d \in \mathbb{N}$ there is $0 \neq \mu \in \mathcal{A}\left(\mathbb{R}^{d}\right)^{\prime}$ such that $T_{\mu}: \mathcal{B}\left(\mathbb{R}^{d}\right) \rightarrow \mathcal{B}\left(\mathbb{R}^{d}\right)$ is not surjective. 
Proof. We reason by contradiction, assuming that there is $d \in \mathbb{N}$ such that $T_{\mu}$ : $\mathcal{B}\left(\mathbb{R}^{d}\right) \rightarrow \mathcal{B}\left(\mathbb{R}^{d}\right)$ is surjective for any $0 \neq \mu \in \mathcal{A}\left(\mathbb{R}^{d}\right)^{\prime}$.

a) We may assume w.l.o.g. that $d=1$.

Proof: Since $T_{\mu}$ is surjective on $\mathcal{B}\left(\mathbb{R}^{d}\right)$ for any $0 \neq \mu \in \mathcal{A}\left(\mathbb{R}^{d}\right)^{\prime}$ this also holds for $\mu:=$ $\nu\left(x_{1}\right) \otimes \delta\left(x^{\prime}\right)$ where $x=\left(x_{1}, x^{\prime}\right) \in \mathbb{R} \times \mathbb{R}^{d-1}$ and $0 \neq \nu \in \mathcal{A}(\mathbb{R})^{\prime}$. Hence, $\widehat{\mu}(z)=\widehat{\nu}\left(z_{1}\right)$ satisfies $(S)$ (on $\mathbb{C}^{d}$ ) by the Main Theorem and therefore $\widehat{\nu}\left(z_{1}\right)$ satisfies $(S)$ (on $\mathbb{C}$ ): in fact, we may apply $(S)$ for $t:=\left(t_{1}, 0\right)$ and notice that $\zeta$ then satisfies $|\zeta| \leq\left|\zeta_{1}\right| /(1-\delta)$. Thus, $T_{\nu}$ is surjective on $\mathcal{B}(\mathbb{R})$ for any $0 \neq \nu \in \mathcal{A}(\mathbb{R})^{\prime}$ by the Main Theorem.

b) Let $T_{\mu}$ be surjective on $\mathcal{B}(\mathbb{R})$ for any $0 \neq \mu \in \mathcal{A}(\mathbb{R})^{\prime}$. Then

$$
\text { any } 0 \neq f \in \mathcal{F}\left(\mathcal{A}([-1,1])^{\prime}\right)=: E \text { satisfies }(S)
$$

by the Main Theorem, where

$$
E=\left\{g \in \mathcal{H}(\mathbb{C})\left|\forall \delta>0: p_{\delta}(g):=\sup _{\mathbb{C}}\right| g(z) \mid e^{-|\Im(z)|-\delta|z|}<\infty\right\}
$$

by the Paley-Wiener theorem (see e.g. 8]).

Let $E_{1}:=\{g \in E \mid g(0)=1\} . E_{1}$ is a complete metrizable space since $E_{1}$ is a closed subset of the Fréchet space $E$.

For any $\eta>0$ there are $g \in E_{1}, \varepsilon>0$ and $C_{1}>1$ such that for any $f \in E_{1}$ with $p_{\varepsilon}(f-g)<\varepsilon$ we have: for any $t \in \mathbb{R}$ with $|t| \geq C_{1}$ there is $\zeta \in \mathbb{C}$ such that

$$
|\zeta-t| \leq \eta|t| \quad \text { and } \quad|f(\zeta)| \geq e^{-\eta|\zeta|}
$$

Proof: For fixed $\eta>0$ let

$$
\left.S_{k}:=\left\{f \in E_{1} \mid f \text { satisfies } 18\right) \text { for } C_{1}:=k\right\} .
$$

$S_{k}$ is closed in $E_{1}$ (since the topology of $E_{1}$ is stronger than locally uniform convergence) and $E_{1}=\bigcup_{k \in \mathbb{N}} S_{k}$ by 17 since $0 \notin E_{1}$. Hence, there is $k_{0}$ by Baire's theorem such that $S_{k_{0}}$ has an interior point. This proves the claim.

c) In the following we will fix $0<\eta<1 / 4$ such that

$$
4 \pi \eta \leq-\ln (\pi \eta) \quad \text { and } \quad|\sin (z)| \leq 2 \text { if }|z-\pi / 2| \leq \eta \pi / 2
$$

and choose $g \in E_{1}, \varepsilon>0$ and $C_{1}>1$ by b).

We may suppose that $g$ is a polynomial. In fact, the polynomials are dense in $E$ (notice that the set $\left\{\delta^{(j)} \mid j \in \mathbb{N}_{0}\right\}$ is total in $\mathcal{A}([-1,1])_{b}^{\prime}$ and that the Fourier transformation is a topological isomorphism from $\mathcal{A}([-1,1])_{b}^{\prime}$ onto $\left.E\right)$. Thus, the polynomials $P$ with $P(0)=1$ are also dense in $E_{1}$.

Let $h_{n}(z):=(\cos (z / n))^{n}$. Then $h_{n}(0)=1$ and

$$
\left|h_{n}(z)\right| \leq e^{|\Im(z)|} \quad \text { for any } n \in \mathbb{N}
$$

and hence $h_{n} \in E_{1}$. Moreover,

$$
h_{n} \rightarrow 1 \quad \text { in } E_{1} .
$$

Proof: Fix $1 \geq \gamma>0$ and let $|z| \geq A:=\frac{1}{\gamma} \ln (2 / \gamma)$. Then $1 \leq \gamma e^{\gamma|z|} / 2$ and

$$
\left|h_{n}(z)-1\right| \leq e^{|\Im(z)|}+1 \leq \gamma e^{|\Im(z)|+\gamma|z|}
$$


by 20 . For $|z| \leq A$ we get by 19 since $|\cos (z)| \leq e^{|\Im(z)|}$

$$
\begin{aligned}
\left|h_{n}(z)-1\right| & \leq|z| \sup _{|\xi| \leq A}|\cos (\xi / n)|^{n-1}|\sin (\xi / n)| \\
& \leq A e^{A} \sup _{|\zeta| \leq A / n}|\sin (\zeta)| \leq \gamma \leq \gamma e^{|\Im(z)|+\gamma|z|} \quad \text { if } n \text { is large. }
\end{aligned}
$$

d) Since $g$ is a polynomial in $E_{1}$ and $h_{n} \in E_{1}$, also $h_{n} g \in E_{1}$. 21 implies that for any $\varepsilon>0$ there is $B_{\varepsilon}$ such that

$$
\left|g(z)-h_{n}(z) g(z)\right| \leq B_{\varepsilon} e^{\varepsilon|z| / 2}\left|h_{n}(z)-1\right| \leq \varepsilon e^{|\Im(z)|+\varepsilon|z|} \quad \text { if } n \in \mathbb{N} \text { is large. }
$$

Since $h_{n} g \in E_{1}$ we thus get by $(22)$ and $(18)$ for large $n$ : for any $t \in \mathbb{R}$ with $|t| \geq C_{1}$ there is $\zeta \in \mathbb{C}$ such that $|\zeta-t| \leq \eta|t|$ and

$$
e^{-\eta|\zeta|} \leq\left|h_{n}(\zeta) g(\zeta)\right| \leq B_{\eta} e^{\eta|\zeta|}\left|h_{n}(\zeta)\right|
$$

and hence

$$
\left|h_{n}(\zeta)\right| \geq e^{-2 \eta|\zeta|} / B_{\eta}
$$

e) Let $t_{n}:=\pi n / 2$ and let $\left|\zeta-t_{n}\right| \leq \eta\left|t_{n}\right|=\eta \pi n / 2$. Then $\left|\zeta / n-t_{n} / n\right| \leq \eta \pi / 2$ and therefore

$$
\begin{aligned}
\left|h_{n}(\zeta)\right| & =|\cos (\zeta / n)|^{n}=\left|\cos (\zeta / n)-\cos \left(t_{n} / n\right)\right|^{n} \\
& \leq 2^{n}\left|\zeta / n-t_{n} / n\right|^{n} \leq(\eta \pi)^{n} \\
& \leq(\eta \pi)^{n / 2} e^{-2 \eta \pi n} \leq e^{-2 \eta|\zeta|} /\left(2 B_{\eta}\right) \quad \text { if } n \text { is large }
\end{aligned}
$$

by 19,24 contradicts 23 .

\section{References}

[1] J. Bonet, A. Galbis and R. Meise, On the range of convolution operators on nonquasianalytic ultradifferentiable functions, Studia Math. 126 (1997), 171-198.

[2] R. W. Braun, R. Meise and D. Vogt, Application of the projective limit functor to convolution and partial differential equations, in: Advances in the Theory of Fréchet Spaces, T. Terzioglu (ed.), NATO ASI Series C, Vol 287, Kluwer, Dordrecht, 1989, 29-46.

[3] R. W. Braun, R. Meise and D. Vogt, Existence of fundamental solutions and surjectivity of convolution operators on classes of ultra-differentiable functions, Proc. London Math. Soc. 61 (1990), 344-370.

[4] C.-C. Chou, La transformation de Fourier complexe et l'équation de convolution, LN Math. 325, Springer, Berlin, 1973.

[5] L. Ehrenpreis, Solution of some problems of division IV, Amer. J. Math. 82 (1960), 522588.

[6] O. v. Grudzinski, Slowly decreasing entire functions and convolution equations, in: Banach Center Publ. 10 (1983), 169-184.

[7] L. Hörmander, On the range of convolution operators, Ann. of Math. 76 (1962), 148-170.

[8] L. Hörmander, An Introduction to Complex Analysis in Several Variables, Van Nostrand, Princeton NJ, 1966.

[9] L. Hörmander, Convolution equations in convex domains, Inventiones Math. 4 (1968), 306-317. 
[10] A. Kaneko, Introduction to Hyperfunctions, Kluwer, Dordrecht, 1988.

[11] T. Kawai, On the theory of Fourier hyperfunctions and its applications to partial differential equations with constant coefficients, J. Fac. Sci. Univ. Tokyo, Sec. IA 17 (1970), 467-517.

[12] M. Langenbruch, Hyperfunction fundamental solutions of surjective convolution operators on real analytic functions, J. Functional Anal. 131 (1995), 78-93.

[13] M. Langenbruch, Convolution operators on Fourier hyperfunctions, J. Generalized Funct., to appear.

[14] M. Langenbruch, Division problems for Fourier ultra-hyperfunctions, Bull. Belg. Math. Soc. Simon Stevin 14 (2007), 521-530.

[15] B. Malgrange, Existence et approximation des solutions des équations aux dérivées partielles et des équations de convolution, Ann. Inst. Fourier (Grenoble) 6 (1955-56), 271-355.

[16] R. Meise, B. A. Taylor and D. Vogt, Equivalence of slowly decreasing conditions and local Fourier expansions, Indiana Univ. Math. J. 36 (1987), 729-756.

[17] R. Meise and D. Vogt, Introduction to Functional Analysis, Clarendon Press, Oxford, 1997.

[18] Y. Okada, Solvability of convolution operators, Publ. RIMS Kyoto Univ. 30 (1994), 167-190.

[19] Y. Saburi, Fundamental properties of modified Fourier hyperfunctions, Tokyo J. Math. 10 (1985), 231-273.

[20] C. Walter, Faltungsoperatoren auf modifizierten Fourierhyperfunktionen, Diploma thesis, Oldenburg, 2007. 
